\title{
Democracy and Foreign Education
}

April 2008

\author{
Antonio Spilimbergo \\ Research Department, IMF; CEPR; WDI
}

Appendix 
This appendix provides background material for the paper "Democracy and Foreign Education." The appendix is organized as follows:

1) Tables A1a to A2b present the basic regression using two alternative measures of democracy:

a) the composite polity II index from the Polity IV dataset, which is the difference between the Polity's democracy and autocracy indices. ${ }^{1}$ Polity IV dataset contains coded annual information on regime and authority characteristics for all independent states (with greater than 500,000 total population) in the global state system and covers the years 1800-2003.

b) the dichotomous democracy index proposed by Przeworski, Alvarez, Cheibub, and Limongi (1990) and extended by Boix and Rosato (2001); a key aspect of this index is that a country is not considered a democracy unless a political party has lost power. In order to make the results easier to interpret, I normalize the indices so that 1 corresponds to the maximum degree of democracy and 0 to the least.

2) Table A3 presents alternative specifications for students using the Przeworski and the polity II indices

3) Table A4 presents the standard regression without the lagged dependent variable. In this case OLS estimators are consistent

4) Tables A5a, A5b, A5c present the standard regressions controlling for domestic educational attainment.

5) Table A6 presents regressions using two sub-samples: one for the period before 1985, the other for the period post 1985

6) Table A7 presents regressions using two sub-samples: one for countries with a population of less than 2 million, the other for countries with population of more than 2 million.

7) Table A8 presents the education of world leaders in 1990.

8) Figures A1, A2, A3 present the evolution of democracy at home and in host countries for Argentina, Iran, and Korea.

9) Table A9 presents the correspondence between country names and three-letter names used in Figure A4.

10) Figure A4 shows the correlation between changes in democracy at home and in host countries.

1 The data are available from the Polity IV's website (http://www.cidcm.umd.edu/inscr/polity/). Note that the name of the variable is polity II in the dataset and throughout the paper. See Marshall and Jaggers (2006) for details on how the variable was constructed. 
Table A1a. Dependent Variable: Polity2 - Levels

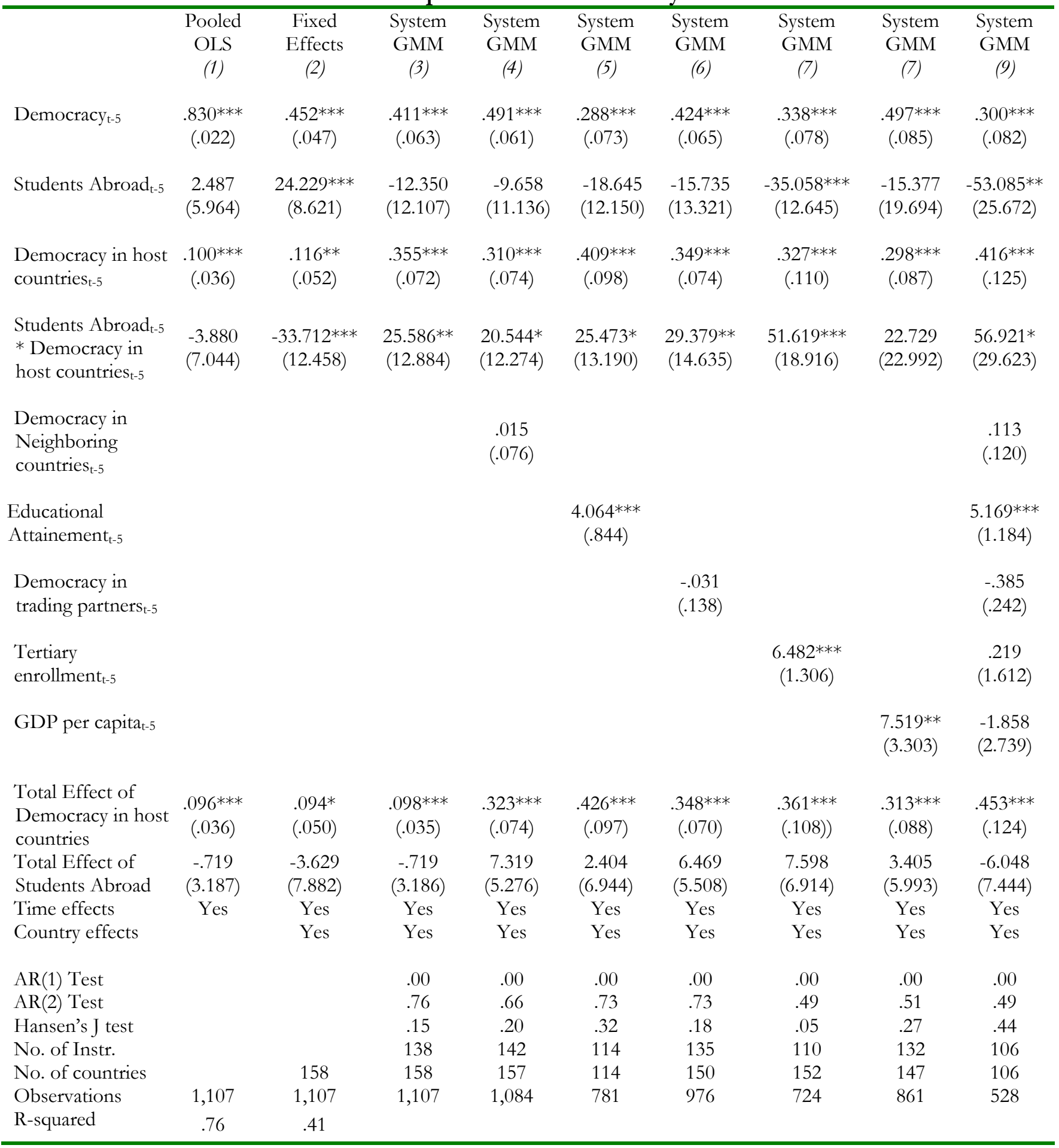

* significant at $10 \%$; ** significant at $5 \%$, *** significant at $1 \%$. Robust standard errors clustered by country in parentheses. AR(1) and AR(2) are Arellano-Bond test for serial correlations. The sample is an unbalanced panel, comprising data at five year interval between 1960 and 2000. Students abroad, democracy in host countries, and all other control variables are treated as predetermined and are instrumented for using their own first to third lags. The total effect of democracy in host countries is calculated summing the coefficient on 'democracy in host countries' and on the interaction between 'students abroad' and 'democracy in host countries', evaluated at average level of students abroad. The total effect of students is calculated summing the coefficient on `students abroad' and on the interaction between `students abroad' and 'democracy in host countries', evaluated at average democracy in host countries. 
Table A1b. Dependent Variable: Polity2 - Differences

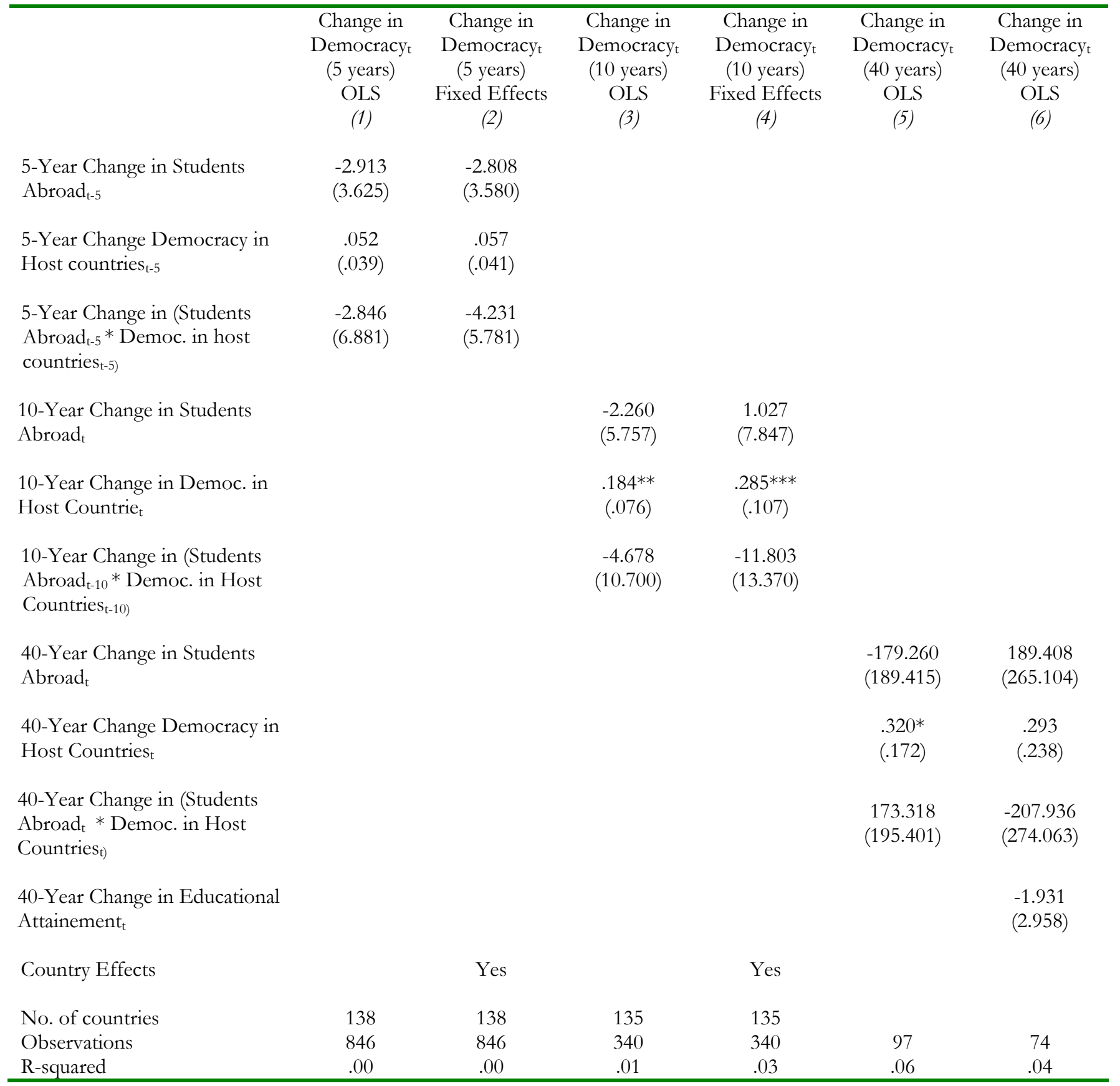

$*$ significant at $10 \%$; $* *$ significant at $5 \%, * * *$ significant at $1 \%$. Robust standard errors clustered by country in parentheses. The sample is an unbalanced panel, comprising data between 1955 and 2000. 
Table A2a. Dependent Variable: Przeworski et al. Democracy Index - Levels

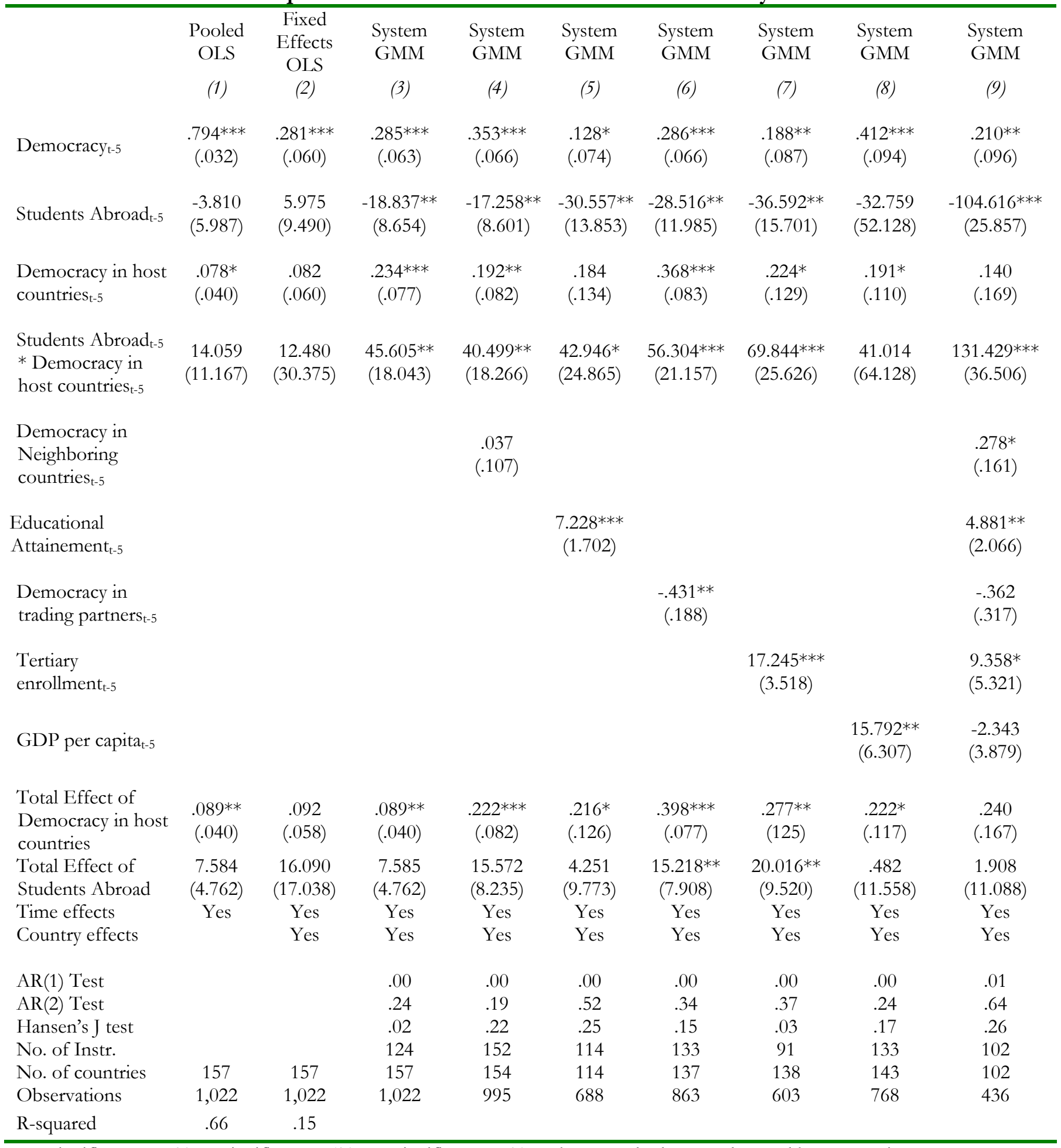

* significant at 10\%; ** significant at 5\%, *** significant at $1 \%$. Robust standard errors clustered by country in parentheses. AR(1) and AR(2) are Arellano-Bond test for serial correlations. The sample is an unbalanced panel, comprising data at five year interval between 1960 and 2000. Students abroad, democracy in host countries, and all other control variables are treated as predetermined and are instrumented for using their own first to third lags. The total effect of democracy in host countries is calculated summing the coefficient on 'democracy in host countries' and on the interaction between 'students abroad' and 'democracy in host countries', evaluated at average level of students abroad. The total effect of students is calculated summing the coefficient on 'students abroad' and on the interaction between 'students abroad' and 'democracy in host countries', evaluated at average democracy in host countries. 
Table A2b. Dependent Variable: Przeworski et al. Democracy Index - Differences

\begin{tabular}{|c|c|c|c|c|c|c|}
\hline & $\begin{array}{l}\text { Change in } \\
\text { Democracyt } \\
\text { (5 years) } \\
\text { OLS } \\
\text { (1) }\end{array}$ & $\begin{array}{l}\text { Change in } \\
\text { Democracy }{ }_{t} \\
(5 \text { years }) \\
\text { Fixed Effects } \\
\text { (2) }\end{array}$ & $\begin{array}{c}\text { Change in } \\
\text { Democracy } \mathrm{t} \\
\text { (10 years) } \\
\text { OLS } \\
\text { (3) }\end{array}$ & $\begin{array}{l}\text { Change in } \\
\text { Democracy }{ }_{t} \\
\text { (10 years) } \\
\text { Fixed Effects } \\
\text { (4) }\end{array}$ & $\begin{array}{c}\text { Change in } \\
\text { Democracyt } \\
\text { (40 years) } \\
\text { OLS } \\
\text { (5) }\end{array}$ & $\begin{array}{l}\text { Change in } \\
\text { Democracyt } \\
\text { (40 years) } \\
\text { OLS } \\
\text { (6) }\end{array}$ \\
\hline $\begin{array}{l}\text { 5-Year Change in Students } \\
\text { Abroad }_{\mathrm{t}-5}\end{array}$ & $\begin{array}{l}21.296 \\
(21.635)\end{array}$ & $\begin{array}{l}22.998 \\
(22.022)\end{array}$ & & & & \\
\hline $\begin{array}{l}5 \text {-Year Change Democracy in } \\
\text { Host countries } t-5\end{array}$ & $\begin{array}{c}.036 \\
(.064)\end{array}$ & $\begin{array}{c}.046 \\
(.069)\end{array}$ & & & & \\
\hline $\begin{array}{l}\text { 5-Year Change in (Students } \\
\text { Abroad }_{\mathrm{t}-5} * \text { Democ. in host } \\
\text { countries }_{\mathrm{t}-5)}\end{array}$ & $\begin{array}{l}-12.258 \\
(14.066)\end{array}$ & $\begin{array}{l}-12.880 \\
(14.442)\end{array}$ & & & & \\
\hline $\begin{array}{l}\text { 10-Year Change in Students } \\
\text { Abroad }_{t}\end{array}$ & & & $\begin{array}{l}3.020 \\
(6.592)\end{array}$ & $\begin{array}{c}12.684 \\
(10.782)\end{array}$ & & \\
\hline $\begin{array}{l}\text { 10-Year Change in Democ. in } \\
\text { Host Countrie }_{t}\end{array}$ & & & $\begin{array}{c}.047 \\
(.112)\end{array}$ & $\begin{array}{l}.209 \\
(.181)\end{array}$ & & \\
\hline $\begin{array}{l}\text { 10-Year Change in (Students } \\
\text { Abroad }_{\mathrm{t}-10} * \text { Democ. in Host } \\
\text { Countries }_{\mathrm{t}-10)}\end{array}$ & & & $\begin{array}{c}11.734 \\
(15.306)\end{array}$ & $\begin{array}{c}5.494 \\
(20.419)\end{array}$ & & \\
\hline $\begin{array}{l}\text { 35-Year Change in Students } \\
\text { Abroad }_{t}\end{array}$ & & & & & $\begin{array}{c}-238.231 * * * \\
(88.980)\end{array}$ & $\begin{array}{l}-120.223 \\
(126.345)\end{array}$ \\
\hline $\begin{array}{l}\text { 35-Year Change Democracy in } \\
\text { Host Countries }_{t}\end{array}$ & & & & & $\begin{array}{l}.466^{* *} \\
(.182)\end{array}$ & $\begin{array}{c}.413 \\
(.282)\end{array}$ \\
\hline $\begin{array}{l}\text { 35-Year Change in (Students } \\
\text { Abroad }_{\mathrm{t}} * \text { Democ. in Host } \\
\left.\text { Countries }_{\mathrm{t}}\right)\end{array}$ & & & & & $\begin{array}{l}251.348^{* * * *} \\
\quad(90.284)\end{array}$ & $\begin{array}{c}149.662 \\
(130.489)\end{array}$ \\
\hline $\begin{array}{l}\text { 35-Year Change in Education } \\
\text { Attainement }\end{array}$ & & & & & & $\begin{array}{l}-3.973 \\
(3.498)\end{array}$ \\
\hline Country Effects & & Yes & & Yes & & \\
\hline No. of countries & 157 & 157 & 146 & 146 & & \\
\hline Observations & 764 & 764 & 361 & 361 & 103 & 78 \\
\hline R-squared & .00 & .00 & .01 & .02 & .08 & .07 \\
\hline
\end{tabular}

* significant at $10 \%$; ** significant at 5\%,*** significant at $1 \%$. Robust standard errors clustered by country in parentheses. The sample is an unbalanced panel, comprising data between 1955 and 2000. 


\section{Table A3. Alternative Specification of the student variables Dependent variable: Democracy indices}

\begin{tabular}{|c|c|c|}
\hline & $\begin{array}{l}\text { Polity II } \\
\text { GMM } \\
\text { (3) }\end{array}$ & $\begin{array}{c}\text { Przeworski } \\
\text { et al. } \\
\text { GMM } \\
\text { (5) }\end{array}$ \\
\hline Democracyt-5 & $\begin{array}{c}.371^{* * *} \\
(.078)\end{array}$ & $\begin{array}{l}.164 * * \\
(.067)\end{array}$ \\
\hline $\begin{array}{l}\text { Students to democratic } \\
\text { countriest-5 }\end{array}$ & $\begin{array}{l}28.924^{*} \\
(17.176)\end{array}$ & $\begin{array}{c}40.841^{* * *} \\
(11.830)\end{array}$ \\
\hline $\begin{array}{l}\text { Students to NON democratic } \\
\text { countriest-5 }\end{array}$ & $\begin{array}{l}-12.257 \\
(18.199)\end{array}$ & $\begin{array}{c}-48.038^{* * *} \\
(13.484)\end{array}$ \\
\hline Time effects & Yes & Yes \\
\hline Country effects & Yes & Yes \\
\hline AR(1) Test & .00 & .00 \\
\hline AR(2) Test & .74 & .48 \\
\hline Hansen's J test & .66 & .14 \\
\hline No. of Instr. & 106 & 95 \\
\hline No. of countries & 158 & 157 \\
\hline Observations & 1,099 & 1,017 \\
\hline
\end{tabular}

* significant at 10\%; ** significant at 5\%, *** significant at 1\%. Robust standard errors clustered by country in parentheses. The sample is an unbalanced panel, comprising data between 1960 and 2000. Students abroad are treated as predetermined and are instrumented for using their own first to third lags. Instrumental variables are described in the text. 
Table A4. Regression without lag dependent variable Dependent Variable: Freedom House Index of Political Freedom.

\begin{tabular}{|c|c|c|c|}
\hline & $\begin{array}{l}\text { Pooled } \\
\text { OLS } \\
\text { (1) }\end{array}$ & $\begin{array}{c}\text { Fixed Effects } \\
\text { OLS } \\
\text { (2) }\end{array}$ & $\begin{array}{c}\text { Fixed Effects } \\
\text { OLS } \\
\text { (3) }\end{array}$ \\
\hline Students Abroad ${ }_{t-5}$ & $\begin{array}{c}12.295 \\
(26.537)\end{array}$ & $\begin{array}{c}9.921 \\
(15.018)\end{array}$ & $\begin{array}{l}30.169 \\
(33.996)\end{array}$ \\
\hline Democracy in host countriest -5 & $\begin{array}{l}.775^{* * *} \\
(.081)\end{array}$ & $\begin{array}{r}.264 * * * \\
(.055)\end{array}$ & $\begin{array}{r}.438^{* * *} \\
(.115)\end{array}$ \\
\hline $\begin{array}{l}\text { Students } \text { Abroad }_{t-5} * \text { Democracy in host } \\
\text { countries }_{t-5}\end{array}$ & $\begin{array}{c}23.824 \\
(29.312)\end{array}$ & $\begin{array}{l}-13.531 \\
(17.995)\end{array}$ & $\begin{array}{r}-64.480^{*} \\
(38.541)\end{array}$ \\
\hline Democracy in Neighboring countries $t_{t-5}$ & & & $\begin{array}{l}-.328 \\
(2.361)\end{array}$ \\
\hline Educational Attainement $t_{t-5}$ & & & $\begin{array}{l}-.262 \\
(.191)\end{array}$ \\
\hline Democracy in trading partners $t_{-5}$ & & & $\begin{array}{l}.643 \\
(1.712)\end{array}$ \\
\hline $\begin{array}{l}\text { Tertiary } \\
\text { enrollment }-5\end{array}$ & & & $\begin{array}{l}.162 \\
(6.109)\end{array}$ \\
\hline GDP per capitat-5 & & & $\begin{array}{l}.206^{*} \\
(.104)\end{array}$ \\
\hline Total Effect of Democracy in host countries & $\begin{array}{l}.799 * * * \\
(.078)\end{array}$ & $\begin{array}{l}.250^{* * *} \\
(.050)\end{array}$ & $\begin{array}{l}.372^{* * *} \\
(.113)\end{array}$ \\
\hline Total Effect of Students Abroad & $\begin{array}{c}32.252^{* * *} \\
(5.518)\end{array}$ & $\begin{array}{l}-1.413 \\
(3.967)\end{array}$ & $\begin{array}{l}-23.844 * * \\
(9.84)\end{array}$ \\
\hline Time effects & Yes & Yes & Yes \\
\hline Country effects & & Yes & Yes \\
\hline $\begin{array}{l}\text { No. of countries } \\
\text { Observations }\end{array}$ & $\begin{array}{c}183 \\
1,393\end{array}$ & $\begin{array}{c}183 \\
1,393\end{array}$ & $\begin{array}{l}108 \\
614\end{array}$ \\
\hline R-squared & .24 & .12 & .16 \\
\hline
\end{tabular}

* significant at $10 \%$; ** significant at $5 \%, * * *$ significant at $1 \%$. Robust standard errors clustered by country in parentheses. The sample is an unbalanced panel, comprising data at five year interval between 1960 and 2000. The total effect of democracy in host countries is calculated summing the coefficient on 'democracy in host countries' and on the interaction between 'students abroad' and 'democracy in host countries', evaluated at average level of students abroad. The total effect of students is calculated summing the coefficient on `students abroad' and on the interaction between 'students abroad' and 'democracy in host countries', evaluated at average democracy in host countries. 
Table A5a. Dependent Variable: Freedom House Index of Political Freedom (controlling for domestic education)

\begin{tabular}{|c|c|c|c|c|c|c|c|}
\hline & $\begin{array}{l}\text { Pooled } \\
\text { OLS } \\
\text { (1) }\end{array}$ & $\begin{array}{l}\text { Fixed } \\
\text { Effects } \\
\text { OLS } \\
\text { (2) }\end{array}$ & $\begin{array}{c}\text { System } \\
\text { GMM } \\
\text { (3) }\end{array}$ & $\begin{array}{c}\text { System } \\
\text { GMM } \\
\text { (4) }\end{array}$ & $\begin{array}{l}\text { System } \\
\text { GMM }\end{array}$ & $\begin{array}{l}\text { System } \\
\text { GMM }\end{array}$ & $\begin{array}{c}\text { System } \\
\text { GMM } \\
\text { (7) }\end{array}$ \\
\hline Democracyt-5 & $\begin{array}{c}0.774 * * * \\
(0.032)\end{array}$ & $\begin{array}{c}.353^{* * *} \\
(.050)\end{array}$ & $\begin{array}{c}.403^{* * *} \\
(.069)\end{array}$ & $\begin{array}{c}.427 * * * \\
(.066)\end{array}$ & $\begin{array}{l}.228^{* * *} \\
(.073)\end{array}$ & $\begin{array}{c}.446^{* * *} \\
(.067)\end{array}$ & $\begin{array}{r}.330 * * * \\
(.070)\end{array}$ \\
\hline $\begin{array}{l}\text { Students } \\
\text { Abroad }_{t-5}\end{array}$ & $\begin{array}{c}6.138 \\
(9.247)\end{array}$ & $\begin{array}{l}35.432 \\
26.337\end{array}$ & $\begin{array}{l}-15.462 \\
(15.272)\end{array}$ & $\begin{array}{c}-5.007 \\
(15.579)\end{array}$ & $\begin{array}{c}-.406 \\
(15.248)\end{array}$ & $\begin{array}{c}3.622 \\
(16.701)\end{array}$ & $\begin{array}{r}-15.006 \\
24.194\end{array}$ \\
\hline $\begin{array}{l}\text { Democracy in } \\
\text { host countriest }-5\end{array}$ & $\begin{array}{l}0.115^{* *} \\
(0.052)\end{array}$ & $\begin{array}{c}0.241 * * * \\
(0.075)\end{array}$ & $\begin{array}{c}0.257 * * * \\
(0.099)\end{array}$ & $\begin{array}{c}0.272^{* * *} \\
(0.096)\end{array}$ & $\begin{array}{c}0.392^{* * * *} \\
(0.107)\end{array}$ & $\begin{array}{l}.289 * * * \\
(.088)\end{array}$ & $\begin{array}{l}.236^{*} \\
(.133)\end{array}$ \\
\hline $\begin{array}{l}\text { Students Abroad } \mathrm{t}_{\mathrm{t}-5} \\
* \text { Democracy in } \\
\text { host countries } \mathrm{t}-5\end{array}$ & $\begin{array}{c}-2.749 \\
(10.745)\end{array}$ & $\begin{array}{c}-56.848^{* *} \\
(28.276)\end{array}$ & $\begin{array}{c}36.875^{*} \\
21.133\end{array}$ & $\begin{array}{c}22.486 \\
(20.686)\end{array}$ & $\begin{array}{c}4.540 \\
(20.940)\end{array}$ & $\begin{array}{c}6.938 \\
(22.444)\end{array}$ & $\begin{array}{l}34.730 \\
(29.325)\end{array}$ \\
\hline $\begin{array}{l}\text { Tertiary } \\
\text { enrollment } t_{t-5}\end{array}$ & $\begin{array}{c}2.263 * * * \\
(0.491)\end{array}$ & $\begin{array}{l}-0.755 \\
(1.027)\end{array}$ & $\begin{array}{c}6.741^{* * *} \\
1.032\end{array}$ & $\begin{array}{c}6.231 * * * \\
(.965)\end{array}$ & $\begin{array}{c}0.603 \\
(1.384)\end{array}$ & $\begin{array}{l}5.980^{* * *} \\
(.971)\end{array}$ & $\begin{array}{c}6.882^{* * * *} \\
(1.189)\end{array}$ \\
\hline $\begin{array}{l}\text { Democracy in } \\
\text { Neighboring } \\
\text { countries }_{t-5}\end{array}$ & & & & $\begin{array}{c}.085 \\
(.083)\end{array}$ & & & \\
\hline $\begin{array}{l}\text { Educational } \\
\text { Attainement } t-5\end{array}$ & & & & & $\begin{array}{c}6.155^{* * *} \\
(1.209)\end{array}$ & & \\
\hline $\begin{array}{l}\text { Democracy in } \\
\text { trading partners } \mathrm{t}_{\mathrm{t}-5}\end{array}$ & & & & & & $\begin{array}{l}.059 \\
(.146)\end{array}$ & \\
\hline $\begin{array}{l}\text { GDP per } \\
\text { capita }_{t-5}\end{array}$ & & & & & & & $\begin{array}{l}3.388 \\
(2.522)\end{array}$ \\
\hline $\begin{array}{l}\text { Total Effect of } \\
\text { Democracy in } \\
\text { host countries }\end{array}$ & $\begin{array}{l}.112^{* *} \\
(.050)\end{array}$ & $\begin{array}{l}.182^{* * *} \\
(.070)\end{array}$ & $\begin{array}{c}.295^{* * *} \\
(.095)\end{array}$ & $\begin{array}{c}.295^{* * *} \\
(.090)\end{array}$ & $\begin{array}{c}.396 * * * \\
(.101)\end{array}$ & $\begin{array}{c}.296^{* * *} \\
(.084)\end{array}$ & $\begin{array}{l}.272^{* *} \\
(.126)\end{array}$ \\
\hline $\begin{array}{l}\text { Total Effect of } \\
\text { Students Abroad }\end{array}$ & $\begin{array}{c}3.815 \\
(2.990)\end{array}$ & $\begin{array}{c}-12.592 \\
(6.165)\end{array}$ & $\begin{array}{c}15.689 * * * \\
(5.312)\end{array}$ & $\begin{array}{c}13.988 * * * \\
(5.050)\end{array}$ & $\begin{array}{c}3.429 \\
(6.355)\end{array}$ & $\begin{array}{c}9.483 \\
(6.430)\end{array}$ & $\begin{array}{c}14.332^{* *} \\
(6.207)\end{array}$ \\
\hline Time effects & Yes & Yes & Yes & Yes & Yes & Yes & Yes \\
\hline Country effects & & Yes & Yes & Yes & Yes & Yes & Yes \\
\hline $\begin{array}{l}\operatorname{AR}(1) \text { Test } \\
\operatorname{AR}(2) \text { Test }\end{array}$ & & & $\begin{array}{l}.00 \\
.53\end{array}$ & $\begin{array}{l}.00 \\
.68\end{array}$ & $\begin{array}{l}.00 \\
.92\end{array}$ & $\begin{array}{l}.00 \\
.63\end{array}$ & $\begin{array}{l}.00 \\
.35\end{array}$ \\
\hline Hansen's J test & & & .05 & .06 & .61 & .06 & .06 \\
\hline No. of Instr. & & & 127 & 128 & 128 & 128 & 128 \\
\hline No. of countries & & 163 & 163 & 163 & 114 & 152 & 156 \\
\hline Observations & 877 & 877 & 877 & 874 & 675 & 834 & 794 \\
\hline R-squared & 0.75 & 0.65 & & & & & \\
\hline
\end{tabular}




\section{Table A5b. Dependent Variable: Polity2 - (controlling for domestic education)}

\begin{tabular}{|c|c|c|c|c|c|c|c|}
\hline & $\begin{array}{l}\text { Pooled } \\
\text { OLS } \\
\text { (1) }\end{array}$ & $\begin{array}{l}\text { Fixed } \\
\text { Effects } \\
\text { OLS } \\
\text { (2) }\end{array}$ & $\begin{array}{l}\text { System } \\
\text { GMM } \\
\text { (3) }\end{array}$ & $\begin{array}{l}\text { System } \\
\text { GMM } \\
\text { (4) }\end{array}$ & $\begin{array}{c}\text { System } \\
\text { GMM } \\
\text { (5) }\end{array}$ & $\begin{array}{c}\text { System } \\
\text { GMM } \\
\text { (6) }\end{array}$ & $\begin{array}{c}\text { System } \\
\text { GMM } \\
\text { (7) }\end{array}$ \\
\hline Democracyt-5 & $\begin{array}{l}.763 * * * \\
(.035)\end{array}$ & $\begin{array}{l}.313^{* * *} \\
(.054)\end{array}$ & $\begin{array}{c}.338^{* * *} \\
(.078)\end{array}$ & $\begin{array}{c}.397 * * * \\
(.075)\end{array}$ & $\begin{array}{c}.259 * * * \\
(.087)\end{array}$ & $\begin{array}{c}.370^{* * *} \\
(.079)\end{array}$ & $\begin{array}{c}.379 * * * \\
(.079)\end{array}$ \\
\hline $\begin{array}{l}\text { Students } \\
\text { Abroad }_{\mathrm{t}-5}\end{array}$ & $\begin{array}{c}-12.253 \\
(7.574)\end{array}$ & $\begin{array}{l}25.427 \\
(16.028)\end{array}$ & $\begin{array}{c}- \\
35.058^{* * *} \\
(12.645)\end{array}$ & $\begin{array}{c}-27.340^{* *} \\
(12.108)\end{array}$ & $\begin{array}{c}-27.477 * * * \\
(9.021)\end{array}$ & $\begin{array}{c}-45.340^{* * *} \\
(15.833)\end{array}$ & $\begin{array}{r}-30.117 \\
20.315\end{array}$ \\
\hline $\begin{array}{l}\text { Democracy in } \\
\text { host countries }{ }_{t-5}\end{array}$ & $\begin{array}{l}.102 * \\
(.058)\end{array}$ & $\begin{array}{c}.276^{* * *} \\
(.095)\end{array}$ & $\begin{array}{c}.327 * * * \\
(.110)\end{array}$ & $\begin{array}{c}.341^{* * *} \\
(.106)\end{array}$ & $\begin{array}{c}.437 * * * \\
(.126)\end{array}$ & $\begin{array}{c}.381 * * * \\
(.102)\end{array}$ & $\begin{array}{l}.309 * * \\
(.125)\end{array}$ \\
\hline $\begin{array}{l}\text { Students } \\
\text { Abroad }_{\mathrm{t}-5} * \\
\text { Democracy in } \\
\text { host countries } \\
\text { t-5 }\end{array}$ & $\begin{array}{l}12.615 \\
(11.222)\end{array}$ & $\begin{array}{c}-51.428^{* *} \\
(23.127)\end{array}$ & $\begin{array}{c}51.619^{* * *} \\
(18.916)\end{array}$ & $\begin{array}{r}39.587 * * \\
(18.439)\end{array}$ & $\begin{array}{r}32.403 * * \\
(14.571)\end{array}$ & $\begin{array}{c}61.065^{* * *} \\
(21.105)\end{array}$ & $\begin{array}{l}45.177 * \\
(23.987)\end{array}$ \\
\hline $\begin{array}{l}\text { Tertiary } \\
\text { enrollment }{ }_{t-5}\end{array}$ & $\begin{array}{c}2.150^{* * *} \\
(.573)\end{array}$ & $\begin{array}{l}-3.331 * \\
(1.794)\end{array}$ & $\begin{array}{c}6.482^{* * *} \\
(1.306)\end{array}$ & $\begin{array}{c}5.863^{* * *} \\
(1.264)\end{array}$ & $\begin{array}{c}-.091 \\
(1.574)\end{array}$ & $\begin{array}{c}6.082^{* * *} \\
(1.271)\end{array}$ & $\begin{array}{c}7.491 * * * \\
(1.715)\end{array}$ \\
\hline $\begin{array}{l}\text { Democracy in } \\
\text { Neighboring } \\
\text { countries }-5^{-5}\end{array}$ & & & & $\begin{array}{l}-.031 \\
(.095)\end{array}$ & & & \\
\hline $\begin{array}{l}\text { Educational } \\
\text { Attainement } t_{t-5}\end{array}$ & & & & & $\begin{array}{c}5.263^{* * *} \\
(1.266)\end{array}$ & & \\
\hline $\begin{array}{l}\text { Democracy in } \\
\text { trading } \\
\text { partners }_{\mathrm{t}-5}\end{array}$ & & & & & & $\begin{array}{l}-.309 \\
(.200)\end{array}$ & \\
\hline $\begin{array}{l}\text { GDP per } \\
\text { capitat-5 }\end{array}$ & & & & & & & $\begin{array}{l}-1.268 \\
(2.967)\end{array}$ \\
\hline $\begin{array}{l}\text { Total Effect of } \\
\text { Democracy in } \\
\text { host countries } \\
\text { Total Effect of } \\
\text { Students } \\
\text { Abroad }\end{array}$ & $\begin{array}{l}-1.678 \\
(3.898)\end{array}$ & $\begin{array}{c}-17.683^{*} \\
(9.311)\end{array}$ & $\begin{array}{l}.369 * * * \\
(.107)\end{array}$ & $\begin{array}{c}.373 * * * \\
(.102)\end{array}$ & $\begin{array}{c}.463 * * * \\
(.123)\end{array}$ & $\begin{array}{c}.345^{* * *} \\
(.122)\end{array}$ & $\begin{array}{c}.462^{* * *} \\
(.124) \\
\\
-5.369 \\
(7.447)\end{array}$ \\
\hline Time effects & Yes & Yes & Yes & Yes & Yes & Yes & Yes \\
\hline Country effects & & Yes & Yes & Yes & Yes & Yes & Yes \\
\hline $\begin{array}{l}\mathrm{AR}(1) \text { Test } \\
\mathrm{AR}(2) \text { Test }\end{array}$ & & & $\begin{array}{r}.00 \\
49\end{array}$ & $\begin{array}{r}.00 \\
37\end{array}$ & $\begin{array}{r}.00 \\
78\end{array}$ & .00 & .00 \\
\hline $\begin{array}{l}\text { AR(2) Test } \\
\text { Hansen's J test }\end{array}$ & & & $\begin{array}{l}.49 \\
.05\end{array}$ & $\begin{array}{l}.37 \\
.16\end{array}$ & $\begin{array}{l}.78 \\
.80\end{array}$ & $\begin{array}{l}.26 \\
.22\end{array}$ & $\begin{array}{l}.23 \\
.30\end{array}$ \\
\hline No. of Instr. & & & 110 & 131 & 131 & 131 & 131 \\
\hline No. of countries & & 152 & 152 & 152 & 112 & 146 & 145 \\
\hline Observations & 724 & 724 & 724 & 721 & 585 & 699 & 650 \\
\hline R-squared & 0.75 & 0.50 & & & & & \\
\hline
\end{tabular}


Table A5b. Dependent Variable: Przeworski et al. Democracy Index - (controlling for domestic education)

\begin{tabular}{|c|c|c|c|c|c|c|c|}
\hline & $\begin{array}{l}\text { Pooled } \\
\text { OLS } \\
\text { (1) }\end{array}$ & $\begin{array}{c}\text { Fixed } \\
\text { Effects } \\
\text { OLS } \\
\text { (2) }\end{array}$ & $\begin{array}{c}\text { System } \\
\text { GMM } \\
\text { (3) }\end{array}$ & $\begin{array}{c}\text { System } \\
\text { GMM } \\
\text { (4) }\end{array}$ & $\begin{array}{c}\text { System } \\
\text { GMM } \\
\text { (5) }\end{array}$ & $\begin{array}{c}\text { System } \\
\text { GMM } \\
\text { (6) }\end{array}$ & $\begin{array}{c}\text { System } \\
\text { GMM } \\
\text { (7) }\end{array}$ \\
\hline Democracyt -5 & $\begin{array}{l}.709^{* * *} \\
(.046)\end{array}$ & $\begin{array}{l}.204^{* * *} \\
(.075)\end{array}$ & $\begin{array}{l}.188^{* *} \\
(.087)\end{array}$ & $\begin{array}{c}.245^{* * *} \\
(.085)\end{array}$ & $\begin{array}{l}.144 \\
(.090)\end{array}$ & $\begin{array}{l}.187 * * \\
(.087)\end{array}$ & $\begin{array}{l}.213^{* *} \\
(.088)\end{array}$ \\
\hline Students Abroad t -5 & $\begin{array}{l}-12.128 \\
(7.937)\end{array}$ & $\begin{array}{c}22.435 \\
(16.873)\end{array}$ & $\begin{array}{c}-36.592 * * \\
(15.701)\end{array}$ & $\begin{array}{c}-33.266^{* * * *} \\
(14.442)\end{array}$ & $\begin{array}{c}-37.892 * * * \\
(13.984)\end{array}$ & $\begin{array}{c}-54.18^{* * *} \\
(19.45)\end{array}$ & $\begin{array}{c}-131.9 * * * \\
(44.104)\end{array}$ \\
\hline $\begin{array}{l}\text { Democracy in hos } 1 \\
\text { countries }_{\mathrm{t}-5}\end{array}$ & $\begin{array}{c}.107 \\
(.066)\end{array}$ & $\begin{array}{l}.380^{* *} \\
(.147)\end{array}$ & $\begin{array}{l}.224^{*} \\
(.129)\end{array}$ & $\begin{array}{c}.171 \\
(.120)\end{array}$ & $\begin{array}{l}.156 \\
(.159)\end{array}$ & $\begin{array}{c}.384 * * * \\
(.120)\end{array}$ & $\begin{array}{l}-.051 \\
(.159)\end{array}$ \\
\hline $\begin{array}{l}\text { Students Abroad }{ }_{\mathrm{t}-5} \\
* \text { Democracy in } \\
\text { host countries }_{\mathrm{t}-5}\end{array}$ & $\begin{array}{c}27.547 * * \\
(13.458)\end{array}$ & $\begin{array}{c}-51.323^{* *} \\
(20.036)\end{array}$ & $\begin{array}{l}69.844 * * * \\
(25.626)\end{array}$ & $\begin{array}{l}62.916^{* * *} \\
(25.249)\end{array}$ & $\begin{array}{l}51.466^{* * *} \\
(25.162)\end{array}$ & $\begin{array}{c}81.174 * * * \\
(32.359)\end{array}$ & $\begin{array}{c}176.31^{* * *} \\
(54.402)\end{array}$ \\
\hline $\begin{array}{l}\text { Tertiary } \\
\text { enrollment } t_{t-5}\end{array}$ & $\begin{array}{c}6.332^{* * *} \\
(1.447)\end{array}$ & $\begin{array}{c}2.130 \\
(3.938)\end{array}$ & $\begin{array}{c}17.245^{* * *} \\
(3.518)\end{array}$ & $\begin{array}{c}16.442^{* * *} \\
(3.345)\end{array}$ & $\begin{array}{c}6.782 \\
(4.374)\end{array}$ & $\begin{array}{c}16.444 * * * \\
(3.246)\end{array}$ & $\begin{array}{c}18.869 * * * \\
(4.422)\end{array}$ \\
\hline $\begin{array}{l}\text { Democracy in } \\
\text { Neighboring } \\
\text { countries }_{t-5}\end{array}$ & & & & $\begin{array}{l}.150 \\
(.124)\end{array}$ & & & \\
\hline $\begin{array}{l}\text { Educational } \\
\text { Attainement } t_{t-5}\end{array}$ & & & & & $\begin{array}{c}6.829 * * * \\
(2.352)\end{array}$ & & \\
\hline $\begin{array}{l}\text { Democracy in } \\
\text { trading partners }{ }_{t-5}\end{array}$ & & & & & & $\begin{array}{c}-.674 * * * \\
(.228)\end{array}$ & \\
\hline $\begin{array}{l}\text { GDP per } \\
\text { capita }_{t-5}\end{array}$ & & & & & & & $\begin{array}{l}1.225 \\
(4.538)\end{array}$ \\
\hline $\begin{array}{l}\text { Total Effect of } \\
\text { Democracy in } \\
\text { host countries }\end{array}$ & $\begin{array}{l}.133^{* *} \\
(.065)\end{array}$ & $\begin{array}{l}.331^{* *} \\
(.137)\end{array}$ & $\begin{array}{l}.290 * * \\
(.124)\end{array}$ & $\begin{array}{l}.231^{* *} \\
(.115)\end{array}$ & $\begin{array}{c}.205 \\
(.148)\end{array}$ & $\begin{array}{c}.462 * * * \\
(.115)\end{array}$ & $\begin{array}{l}.116 \\
(.156)\end{array}$ \\
\hline $\begin{array}{l}\text { Total Effect of } \\
\text { Students Abroad }\end{array}$ & $\begin{array}{l}10.152^{*} \\
(5.762)\end{array}$ & $\begin{array}{l}-19.086 \\
(18.314)\end{array}$ & $\begin{array}{c}19.898^{* *} \\
(9.489)\end{array}$ & $\begin{array}{l}17.621 * \\
(10.141)\end{array}$ & $\begin{array}{c}3.734 \\
(11.785)\end{array}$ & $\begin{array}{c}11.478 \\
(12.221)\end{array}$ & $\begin{array}{l}10.699 \\
(7.601)\end{array}$ \\
\hline Time effects & Yes & Yes & Yes & Yes & Yes & Yes & Yes \\
\hline Country effects & & Yes & Yes & Yes & Yes & Yes & Yes \\
\hline $\begin{array}{l}\text { AR(1) Test } \\
\text { AR(2) Test }\end{array}$ & & & $\begin{array}{r}.00 \\
37\end{array}$ & $\begin{array}{r}.00 \\
22\end{array}$ & $\begin{array}{l}.01 \\
77\end{array}$ & .00 & $\begin{array}{l}.01 \\
36\end{array}$ \\
\hline Hansen's J test & & & .04 & .07 & .34 & .10 & .11 \\
\hline No. of Instr. & & & 93 & 110 & 108 & 110 & 110 \\
\hline No. of countries & & 138 & 138 & 137 & 110 & 129 & 130 \\
\hline Observations & 603 & 603 & 603 & 600 & 487 & 576 & 534 \\
\hline R-squared & 0.75 & 0.50 & & & & & \\
\hline
\end{tabular}


Table A6. Earlier Versus Later Periods (cut-off 1985)

\begin{tabular}{|c|c|c|c|c|c|c|}
\hline & $\begin{array}{c}\text { Freedom } \\
\text { House } \\
\text { Year } \\
\leq 1985 \\
\text { (1) }\end{array}$ & $\begin{array}{c}\text { Freedom } \\
\text { House } \\
\text { Year } \\
>1985 \\
\text { (2) }\end{array}$ & $\begin{array}{c}\text { Polity II } \\
\text { Year } \\
\leq 1985 \\
\text { (3) }\end{array}$ & $\begin{array}{c}\text { Polity II } \\
\text { Year } \\
>1985 \\
\text { (4) }\end{array}$ & $\begin{array}{l}\text { Przeworski } \\
\text { et al. } \\
\text { Year } \\
\leq 1985 \\
\text { (5) }\end{array}$ & $\begin{array}{c}\text { Przeworski } \\
\text { et al. } \\
\text { Year } \\
>1985 \\
\text { (6) }\end{array}$ \\
\hline Democracy $_{t-5}$ & $\begin{array}{c}.528^{* * * *} \\
(.064)\end{array}$ & $\begin{array}{c}.644^{* * * *} \\
(.043)\end{array}$ & $\begin{array}{c}.401 * * * \\
(.091)\end{array}$ & $\begin{array}{c}.525^{* * *} \\
(.057)\end{array}$ & $\begin{array}{l}.172 * * \\
(0.084)\end{array}$ & $\begin{array}{c}0.590 * * * \\
(.061)\end{array}$ \\
\hline $\begin{array}{l}\text { Students } \\
\text { Abroad }_{\mathrm{t}-5}\end{array}$ & $\begin{array}{c}-22.946^{* *} \\
(9.941)\end{array}$ & $\begin{array}{c}4.739 \\
(17.856)\end{array}$ & $\begin{array}{l}-19.066 \\
(11.958)\end{array}$ & $\begin{array}{l}-34.188 \\
(20.196)\end{array}$ & $\begin{array}{l}-20.656^{*} \\
(11.623)\end{array}$ & $\begin{array}{l}-24.980 \\
(26.032)\end{array}$ \\
\hline $\begin{array}{l}\text { Democracy in host } \\
\text { countries }_{t-5}\end{array}$ & $\begin{array}{l}.315^{* * *} \\
(.071)\end{array}$ & $\begin{array}{c}.061 \\
(.084)\end{array}$ & $\begin{array}{c}.383^{* * *} \\
(.086)\end{array}$ & $\begin{array}{l}.222^{* *} \\
(.105)\end{array}$ & $\begin{array}{l}.389 * * * \\
(0.086)\end{array}$ & $\begin{array}{l}.072 \\
(.119)\end{array}$ \\
\hline $\begin{array}{l}\text { Students Abroad }{ }_{t-5} * \\
\text { Democracy in host } \\
\text { countries }_{t-5}\end{array}$ & $\begin{array}{c}52.690^{* * * *} \\
(16.305)\end{array}$ & $\begin{array}{c}9.147 \\
(19.340)\end{array}$ & $\begin{array}{l}39.678 * * \\
(15.575)\end{array}$ & $\begin{array}{l}44.768^{*} \\
(23.727)\end{array}$ & $\begin{array}{c}60.147^{* * *} \\
(23.046)\end{array}$ & $\begin{array}{c}39.111 \\
(30.758)\end{array}$ \\
\hline $\begin{array}{l}\text { Time fixed effects } \\
\text { Country fixed } \\
\text { effects }\end{array}$ & $\begin{array}{l}\text { Yes } \\
\text { Yes }\end{array}$ & $\begin{array}{l}\text { Yes } \\
\text { Yes }\end{array}$ & $\begin{array}{l}\text { Yes } \\
\text { Yes }\end{array}$ & $\begin{array}{l}\text { Yes } \\
\text { Yes }\end{array}$ & $\begin{array}{l}\text { Yes } \\
\text { Yes }\end{array}$ & $\begin{array}{l}\text { Yes } \\
\text { Yes }\end{array}$ \\
\hline $\begin{array}{l}\text { AR(1) Test } \\
\text { AR(2) Test } \\
\text { Hansen's J test }\end{array}$ & $\begin{array}{c}0 \\
.97 \\
.01\end{array}$ & $\begin{array}{l}0 \\
.51 \\
0\end{array}$ & $\begin{array}{l}0 \\
.63 \\
.03\end{array}$ & $\begin{array}{c}0 \\
.18 \\
0\end{array}$ & $\begin{array}{c}0 \\
.26 \\
0\end{array}$ & $\begin{array}{l}0 \\
.25 \\
.04\end{array}$ \\
\hline $\begin{array}{l}\text { No. of Instruments } \\
\text { No. of countries } \\
\text { Observations }\end{array}$ & $\begin{array}{c}90 \\
154 \\
707 \\
\end{array}$ & $\begin{array}{c}92 \\
182 \\
672 \\
\end{array}$ & $\begin{array}{c}90 \\
136 \\
685 \\
\end{array}$ & $\begin{array}{c}78 \\
156 \\
422 \\
\end{array}$ & $\begin{array}{c}90 \\
147 \\
719 \\
\end{array}$ & $\begin{array}{c}65 \\
156 \\
303 \\
\end{array}$ \\
\hline
\end{tabular}

* significant at $10 \%$; ** significant at $5 \%, * * *$ significant at $1 \%$. Robust standard errors clustered by country in parentheses. AR(1) and $\mathrm{AR}(2)$ are Arellano-Bond test for serial correlations. The sample is an unbalanced panel, comprising data at five year interval between 1960 and 1985 for specifications (1), (2), and (3), between 1990 and 2005 for specification (2), and between 1990 and 2000 for specifications (4) and (6). Students abroad and democracy in host countries are considered predetermined and are instrumented for using their own first and second lags. 
Table A7. Small Versus Large Countries (cut-off 2 million)

\begin{tabular}{|c|c|c|c|c|c|c|}
\hline & $\begin{array}{l}\text { Freedom } \\
\text { House } \\
\text { Population } \\
<2 \text { mil } \\
\text { (1) }\end{array}$ & $\begin{array}{l}\text { Freedom } \\
\text { House } \\
\text { Population } \\
>2 \text { mil } \\
\text { (2) }\end{array}$ & $\begin{array}{l}\text { Polity II } \\
\text { Population } \\
<2 \text { mil } \\
\text { (3) }\end{array}$ & $\begin{array}{l}\text { Polity II } \\
\text { Population } \\
>2 \text { mil } \\
\text { (4) }\end{array}$ & $\begin{array}{l}\text { Przeworski } \\
\text { et al. } \\
\text { Population } \\
<2 \text { mil } \\
\text { (5) }\end{array}$ & $\begin{array}{l}\text { Przeworski } \\
\text { et al. } \\
\text { Population } \\
>2 \text { mil } \\
\text { (6) }\end{array}$ \\
\hline Democracy $y_{t-5}$ & $\begin{array}{c}.652^{* * * *} \\
(.072)\end{array}$ & $\begin{array}{l}.547 * * * \\
(.052)\end{array}$ & $\begin{array}{c}.766^{* * *} \\
(.085)\end{array}$ & $\begin{array}{c}.439 * * * \\
(.063)\end{array}$ & $\begin{array}{l}.508^{* * *} \\
(.096)\end{array}$ & $\begin{array}{l}.364 * * * \\
(.070)\end{array}$ \\
\hline $\begin{array}{l}\text { Students } \\
\text { Abroad }_{\mathrm{t}-5}\end{array}$ & $\begin{array}{l}-11.936 \\
(10.872)\end{array}$ & $\begin{array}{c}7.463 \\
(16.118)\end{array}$ & $\begin{array}{l}-2.467 \\
(8.472)\end{array}$ & $\begin{array}{l}-20.624 \\
(22.508)\end{array}$ & $\begin{array}{c}-10.512 \\
(6.957)\end{array}$ & $\begin{array}{c}7.542 \\
(19.879)\end{array}$ \\
\hline $\begin{array}{l}\text { Democracy in } \\
\text { host countries }{ }_{t-5}\end{array}$ & $\begin{array}{l}.164 * * \\
(.077)\end{array}$ & $\begin{array}{c}.238^{* * * *} \\
(.064)\end{array}$ & $\begin{array}{c}.091 \\
(.066)\end{array}$ & $\begin{array}{c}.365^{* * *} \\
(.074)\end{array}$ & $\begin{array}{l}.196^{*} \\
(.104)\end{array}$ & $\begin{array}{l}.367 * * * \\
(.087)\end{array}$ \\
\hline $\begin{array}{l}\text { Students } \\
\text { Abroad }_{t-5} * \\
\text { Democracy in } \\
\text { host countries }_{t-5}\end{array}$ & $\begin{array}{l}30.384 * * \\
(14.633)\end{array}$ & $\begin{array}{c}-4.904 \\
(26.627)\end{array}$ & $\begin{array}{c}11.826 \\
(10.079)\end{array}$ & $\begin{array}{c}29.739 \\
(40.415)\end{array}$ & $\begin{array}{c}50.415^{* * *} \\
(11.101)\end{array}$ & $\begin{array}{l}-36.169 \\
(54.140)\end{array}$ \\
\hline $\begin{array}{l}\text { Time fixed } \\
\text { effects }\end{array}$ & Yes & Yes & Yes & Yes & Yes & Yes \\
\hline $\begin{array}{l}\text { Country fixed } \\
\text { effects }\end{array}$ & Yes & Yes & Yes & Yes & Yes & Yes \\
\hline AR(1) Test & .00 & .00 & .01 & .00 & .07 & .00 \\
\hline AR(2) Test & .52 & .78 & .80 & .67 & .04 & .18 \\
\hline Hansen's J test & 1.00 & .38 & 1.00 & .53 & 1.00 & .35 \\
\hline $\begin{array}{l}\text { No. of } \\
\text { Instruments }\end{array}$ & 140 & 155 & 137 & 141 & 117 & 128 \\
\hline $\begin{array}{l}\text { No. of } \\
\text { countries }\end{array}$ & 60 & 180 & 39 & 138 & 55 & 121 \\
\hline Observations & 266 & 1,113 & 160 & 947 & 204 & 818 \\
\hline
\end{tabular}

* significant at $10 \%$; $* *$ significant at $5 \%$, *** significant at $1 \%$. Robust standard errors clustered by country in parentheses. $\operatorname{AR}(1)$ and $\operatorname{AR}(2)$ are Arellano-Bond test for serial correlations. The sample is an unbalanced panel, comprising data at five year interval between 1960 and 2005. Students abroad and democracy in host countries are considered predetermined and are instrumented for using their own first and second lags and their differences. 
Table A8. Education of World Leaders in 1990

\begin{tabular}{|c|c|c|c|c|c|c|}
\hline Country & Last name & First name & Primary & Secondary & Tertiary & Other \\
\hline Angola & Dos Santos & Jose Eduardo & . & . & Russia & . \\
\hline Argentina & Menem & Carlos & Argentina & Argentina & Argentina & . \\
\hline Australia & Hawke & $\begin{array}{l}\text { Robert James } \\
\text { Lee }\end{array}$ & Australia & Australia & Australia & UK \\
\hline Austria & Vranitzky & Franz & Austria & Austria & Austria & \\
\hline Bangladesh & Ershad & $\begin{array}{l}\text { Hossain } \\
\text { Mohammad }\end{array}$ & Bangladesh & Bangladesh & Bangladesh & India \\
\hline Barbados & Sandiford & Lloyd Erskine & . & . & Jamaica & UK \\
\hline Belgium & Martens & Wilfried & . & . & Belgium & . \\
\hline Benin & Kerekou & Mathieu & & & France & \\
\hline Bolivia & Paz (Zamora) & Jaime & Bolivia & Bolivia & Chile & . \\
\hline Brazil & Sarnay & Jose & Brazil & Brazil & Brazil & . \\
\hline Cameroon & Biya & Paul & . & . & France & . \\
\hline Canada & Mulroney & Martin Brian & Canada & Canada & Canada & . \\
\hline Chad & Habre & Hissene & Chad & Chad & France & . \\
\hline Chile & $\begin{array}{l}\text { Pinochet } \\
\text { (Ugarte) }\end{array}$ & Augusto & Chile & Chile & . & Chile \\
\hline China & Xiaoping & Deng & China & China & France & Russia \\
\hline Colombia & Gaviria(Trujillo) & Cesar & . & . & Colombia & . \\
\hline $\begin{array}{l}\text { Congo, } \\
\text { Dem. Rep. }\end{array}$ & Seko & $\begin{array}{l}\text { Mobutu Sese } \\
\text { (Joseph) }\end{array}$ & $\begin{array}{l}\text { Congo, } \\
\text { Dem. Rep. }\end{array}$ & $\begin{array}{l}\text { Congo, } \\
\text { Dem. Rep. }\end{array}$ & . & Belgium \\
\hline $\begin{array}{l}\text { Congo, } \\
\text { Republic of }\end{array}$ & Sassou-Nguesso & Denis & . & . & . & $\begin{array}{l}\text { Algeria } \\
\text { France }\end{array}$ \\
\hline Costa Rica & Sanchez & $\begin{array}{l}\text { Oscar Rafael } \\
\text { de Jesus Arias }\end{array}$ & Costa Rica & Costa Rica & US & Costa Rica \\
\hline $\begin{array}{l}\text { Côte } \\
\text { d'Ivoire }\end{array}$ & $\begin{array}{l}\text { Houphouet- } \\
\text { Boigny }\end{array}$ & Felix & $\begin{array}{l}\text { Côte } \\
\text { d'Ivoire }\end{array}$ & $\begin{array}{l}\text { Côte } \\
\text { d'Ivoire }\end{array}$ & Senegal & . \\
\hline Denmark & Schluter & $\begin{array}{l}\text { Poul } \\
\text { Holmskov }\end{array}$ & . & . & Denmark & Denmark \\
\hline Dominica & Charles & Mary Eugenia & Dominica & Dominica & Canada & $\begin{array}{l}\text { UK } \\
\text { Grenada }\end{array}$ \\
\hline Dominican & Balaguer y & Joaquin & Dominican & Dominican & Dominican & France \\
\hline Republic & Ricardo & Amparo & Republic & Republic & Republic & \\
\hline Ecuador & Borja (Cevallos) & Rodrigo & Ecuador & Ecuador & Ecuador & Ecuador \\
\hline Egypt & Mubarak & Hosni & Egypt & Egypt & Egypt & \\
\hline El Salvador & Burkard & $\begin{array}{l}\text { Alfredo Felix } \\
\text { Cristiani }\end{array}$ & El Salvador & El Salvador & US & \\
\hline Equatorial & Obiang & Teodoro & Equatorial & Equatorial & Spain & \\
\hline Guinea & Nguema & & Guinea & Guinea & & \\
\hline Ethiopia & Haile Mariam & Mengistu & Ethiopia & Ethiopia & Ethiopia & \\
\hline
\end{tabular}

(Continues)

Source: Author's research. The list of leaders is from the list of world leaders from Jones and Olken (2005). The table includes leaders who were in power in 1990. The information was collected by checking biography of each leader. If no information was available a missing value is indicated. Therefore, the numbers are a lower bound for the actual number of foreign-educated leaders. 
Table A8. (Continuation) Education of world leaders in 1990

\begin{tabular}{|c|c|c|c|c|c|c|}
\hline Country & Last name & First name & Primary & Secondary & Tertiary & Other \\
\hline Fiji & Mara & Kamisese & . & . & New Zealand & UK \\
\hline Finland & Koivisto & Mauno Henrik & Finland & Finland & Finland & Finland \\
\hline France & Mitterrand & Francois & France & France & France & . \\
\hline Gabon & Bongo & Omar & Congo, Rep. & Congo, Rep. & . & Chad \\
\hline Gambia, The & Jawara & Sir Dawda & . & . & UK & . \\
\hline Ghana & Rawlings & Jerry & Ghana & Ghana & . & Ghana \\
\hline Greece & Mitsotakis & $\begin{array}{l}\text { Konstantinos } \\
\text { Kiriakou }\end{array}$ & . & . & Greece & . \\
\hline Guatemala & Arevalo & $\begin{array}{l}\text { Marco Vinicio } \\
\text { Cerezo }\end{array}$ & Guatemala & Guatemala & Guatemala & . \\
\hline Guinea & Conte & Lansana & & $\begin{array}{l}\text { Côte } \\
\text { d'Ivoire }\end{array}$ & & \\
\hline Honduras & Romero & . & . & . & US & \\
\hline Hungary & Antall & Jozsef & Hungary & Hungary & Hungary & \\
\hline Iceland & Hermannsson & Steingrimur & Iceland & Iceland & US & US \\
\hline India & Shekhar & Chandra & . & . & . & India \\
\hline Indonesia & Suharto & & . & . & Indonesia & . \\
\hline Iran & Khamenei & $\begin{array}{l}\text { Sayyed } \\
\text { Mohammad } \\
\text { Ali Hoseyn }\end{array}$ & Iran & Iran & Iran & \\
\hline Ireland & Haughey & Charles James & Ireland & Ireland & Ireland & . \\
\hline Israel & Shamir & Yitzhak & . & Poland & Palestine & \\
\hline Italy & Andreotti & Giulio & . & . & Italy & \\
\hline Jamaica & Manley & $\begin{array}{l}\text { Michael } \\
\text { Norman }\end{array}$ & . & . & UK & \\
\hline Japan & Kaifu & Toshiki & . & . & Japan & \\
\hline Jordan & Al-Hashimi & $\begin{array}{l}\text { Hussein ibn } \\
\text { Talal }\end{array}$ & . & UK & UK & \\
\hline Kenya & Moi & Daniel Arap & . & Kenya & Kenya & \\
\hline $\begin{array}{l}\text { Korea, } \\
\text { Republic of } \\
\text { Lesotho }\end{array}$ & $\begin{array}{l}\text { Roh } \\
\text { Lekhanya }\end{array}$ & $\begin{array}{l}\text { Tae Woo } \\
\text { Justin }\end{array}$ & . & $\begin{array}{l}\text { Korea, } \\
\text { Republic of } \\
\text {. }\end{array}$ & $\begin{array}{l}\text { Korea, } \\
\text { Republic of } \\
\text {. }\end{array}$ & \\
\hline Liberia & Doe & Samuel K. & . & . & . & \\
\hline Liberia & Sawyer & Amos & . & . & . & US \\
\hline Luxembourg & Santer & Jacques & . & . & France & France \\
\hline Madagascar & Ratsiraka & Didier & . & Madagascar & France & \\
\hline Malawi & Banda & Hastings $\mathrm{K}$. & . & US & US & UK \\
\hline Malaysia & Mohamad & $\begin{array}{l}\text { Datuk Ser } \\
\text { Mahathir Bin }\end{array}$ & . & . & Malaysia & Singapore \\
\hline Mali & Traore & Moussa & . & Mali & France & . \\
\hline
\end{tabular}


Table A8. (Continuation) Education of World Leaders in 1990

\begin{tabular}{|c|c|c|c|c|c|c|}
\hline Mauritania & Taya & $\begin{array}{l}\text { Maaouya Ould } \\
\text { Sid'ahmed }\end{array}$ & Mauritania & Mauritania & Mauritania & \\
\hline Mauritius & Jugnauth & Aneerood & . & . & UK & . \\
\hline Mexico & de Gortari & Carlos Salinas & . & . & Mexico & US \\
\hline Morocco & Mohammed & $\begin{array}{l}\text { Mawlay al-Hasan II } \\
\text { ibn }\end{array}$ & . & . & Morocco & France \\
\hline Mozambique & Chissano & Joaquim & Mozambique & Mozambique & France & Portugal \\
\hline Namibia & Nujoma & Sam & Namibia & Namibia & & \\
\hline Nepal & Birendra & $\begin{array}{l}\text { Mohan Shumshere } \\
\text { J.B. }\end{array}$ & . & UK & US & Japan \\
\hline Netherlands & Lubbers & $\begin{array}{l}\text { Rudolphus Frans } \\
\text { Marie (Ruud) }\end{array}$ & . & . & Netherlands & . \\
\hline New Zealand & Palmer & $\begin{array}{l}\text { Geoffrey Winston } \\
\text { Russel }\end{array}$ & . & New Zealand & New Zealand & US \\
\hline Nicaragua & Saavedra & Jose Daniel Ortega & Nicaragua & Nicaragua & Nicaragua & . \\
\hline Niger & Seibou & Ali & . & Senegal & Niger & . \\
\hline Nigeria & Babangida & Ibrahim & Nigeria & Nigeria & UK & . \\
\hline Norway & Brundtland & Gro Harlem & . & . & Norway & US \\
\hline Pakistan & Sharif & Nawaz & . & Pakistan & Pakistan & Pakistan \\
\hline Pakistan & Bhutto & Benazir & Pakistan & Pakistan & US & UK \\
\hline Panama & Galimany & $\begin{array}{l}\text { Guillermo David } \\
\text { Endara }\end{array}$ & . & . & Panama & US \\
\hline $\begin{array}{l}\text { Papua New } \\
\text { Guinea }\end{array}$ & Namaliu & Rabbie & . & . & $\begin{array}{l}\text { Papua New } \\
\text { Guinea }\end{array}$ & Canada \\
\hline Paraguay & $\begin{array}{l}\text { Rodriguez } \\
\text { (Pedotti) }\end{array}$ & Andres & . & . & . & . \\
\hline Peru & Garcia (Perez) & Alan & Peru & Peru & Peru & Spain \\
\hline Philippines & Aquino & Corazon & US & US & US & Philippines \\
\hline Poland & Walesa & Lech & Poland & . & . & . \\
\hline Portugal & Silva & Anibal Cavaco & . & . & US & UK \\
\hline Romania & Iliescu & Ion & Romania & Romania & Russia & . \\
\hline Rwanda & Habyarimana & Juvenal & . & . & Zaire & Zaire \\
\hline $\begin{array}{l}\text { São Tomé } \\
\text { and Príncipe }\end{array}$ & Costa & Manuel Pinto Da & . & . & . & . \\
\hline Senegal & Diouf & Abdul & . & . & . & . \\
\hline Seychelles & Rene & France Albert & . & . & UK & UK \\
\hline Sierra Leone & Momoh & Joseph Saidu & . & . & . & $\begin{array}{l}\text { Ghana, } \\
\text { Nigeria,UK }\end{array}$ \\
\hline
\end{tabular}


Table A8. (Continuation)Education of world leaders in 1990

\begin{tabular}{|c|c|c|c|c|c|c|}
\hline Singapore & Lee & Kuan Yew & Singapore & Singapore & Singapore & UK \\
\hline Singapore & Goh & Chok Tong & . & . & Singapore & US \\
\hline Somalia & Barre & $\begin{array}{l}\text { Mohammed } \\
\text { Siad }\end{array}$ & & & & Italy \\
\hline South Africa & de Klerk & F.W. & . & . & South Africa & . \\
\hline Spain & Marquez & $\begin{array}{l}\text { Felipe } \\
\text { Gonzales }\end{array}$ & . & . & Spain & Spain \\
\hline Sri Lanka & Premadasa & Ranasinghe & . & . & Sri Lanka & . \\
\hline $\begin{array}{l}\text { St. Kitts \& } \\
\text { Nevis }\end{array}$ & Simmonds & Kennedy & $\begin{array}{l}\text { St. Kitts \& } \\
\text { Nevis }\end{array}$ & $\begin{array}{l}\text { St. Kitts \& } \\
\text { Nevis }\end{array}$ & Jamaica & Bahamas \\
\hline St. Lucia & Compton & John & . & . & . & UK \\
\hline $\begin{array}{l}\text { St.Vincent \& } \\
\text { Grenadines }\end{array}$ & Mitchell & $\begin{array}{l}\text { James Fitz- } \\
\text { Allen }\end{array}$ & . & . & $\begin{array}{l}\text { Trinidad } \\
\text { \&Tobago }\end{array}$ & Canada \\
\hline Sudan & Al-Bashir & Omar & . & . & . & Egypt \\
\hline Sweden & Carlsson & Ingvar Gosta & Sweden & Sweden & US & . \\
\hline Switzerland & Koller & Arnold & . & . & . & . \\
\hline Syria & al-Assad & $\begin{array}{l}\text { Abu Sulayman } \\
\text { Hafiz }\end{array}$ & Syria & Syria & & Syria \\
\hline $\begin{array}{l}\text { Taiwan, } \\
\text { Province of } \\
\text { China }\end{array}$ & Teng-Hui & Lee & $\begin{array}{l}\text { Taiwan, } \\
\text { Province of } \\
\text { China }\end{array}$ & $\begin{array}{l}\text { Taiwan, } \\
\text { Province of } \\
\text { China }\end{array}$ & Japan & $\begin{array}{l}\text { Taiwan, } \\
\text { Province } \\
\text { of China }\end{array}$ \\
\hline Tanzania & Mwinyi & Ali Hassan & Tanzania & Tanzania & Tanzania & UK \\
\hline Thailand & Choonhavan & Chatichai & Thailand & Thailand & Thailand & US \\
\hline Togo & Eyadema & Gnassingbe & Togo & Togo & . & 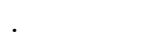 \\
\hline $\begin{array}{l}\text { Trinidad \& } \\
\text { Tobago }\end{array}$ & Robinson & $\begin{array}{l}\text { Arthur } \\
\text { Napoleon }\end{array}$ & $\begin{array}{l}\text { Trinidad \& } \\
\text { Tobago }\end{array}$ & $\begin{array}{l}\text { Trinidad \& } \\
\text { Tobago }\end{array}$ & UK & UK \\
\hline Tunisia & Ali & $\begin{array}{l}\text { Zine El } \\
\text { Abidine Ben }\end{array}$ & . & . & France & US \\
\hline Turkey & Ozal & Turgut & Turkey & Turkey & Turkey & US \\
\hline Uganda & Museveni & Yoweri & Uganda & Uganda & Tanzania & . \\
\hline $\begin{array}{l}\text { United } \\
\text { Kingdom }\end{array}$ & Major & John Roy & UK & UK & . & . \\
\hline $\begin{array}{l}\text { United } \\
\text { Kingdom }\end{array}$ & Thatcher & Margaret Hilda & UK & UK & UK & . \\
\hline United States & Bush & George H. & US & US & US & . \\
\hline Uruguay & Lacalle & Luis & . & . & Uruguay & . \\
\hline Uruguay & $\begin{array}{l}\text { Sanguinetti } \\
\text { (Cairolo) }\end{array}$ & Julio & . & . & Uruguay & . \\
\hline Venezuela & Perez & Carlos Andres & . & Venezuela & Venezuela & . \\
\hline Yemen & al-Hashidi & $\begin{array}{l}\text { Ali 'Abd Allah } \\
\text { Saleh }\end{array}$ & Yemen & Yemen & Yemen & . \\
\hline Zimbabwe & Mugabe & Robert & . & r. & South Africa & UK \\
\hline
\end{tabular}




\section{Figure A1. Argentina}
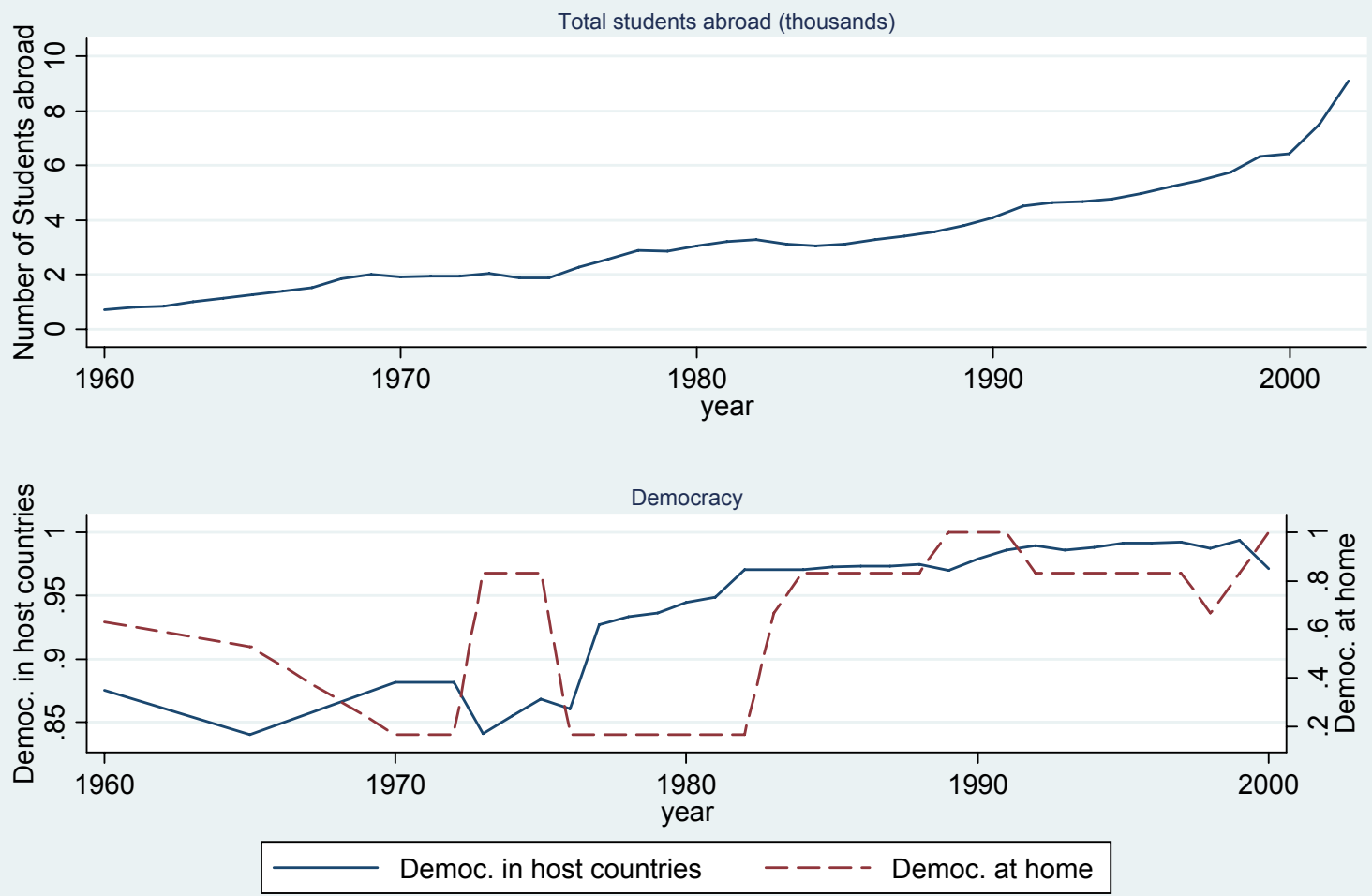

Note: the top panel shows total number of Argentinean students abroad in expressed in thousands. The bottom panel shows the level of democracy in home country and in students' host countries, i.e. the average level of democracy in countries where Argentinean students are. Both indices are constructed using the Freedom House index of democracy normalized so it lies between 0 and 1 . The construction of the data is described in the data appendix. 


\section{Figure A2. Iran}
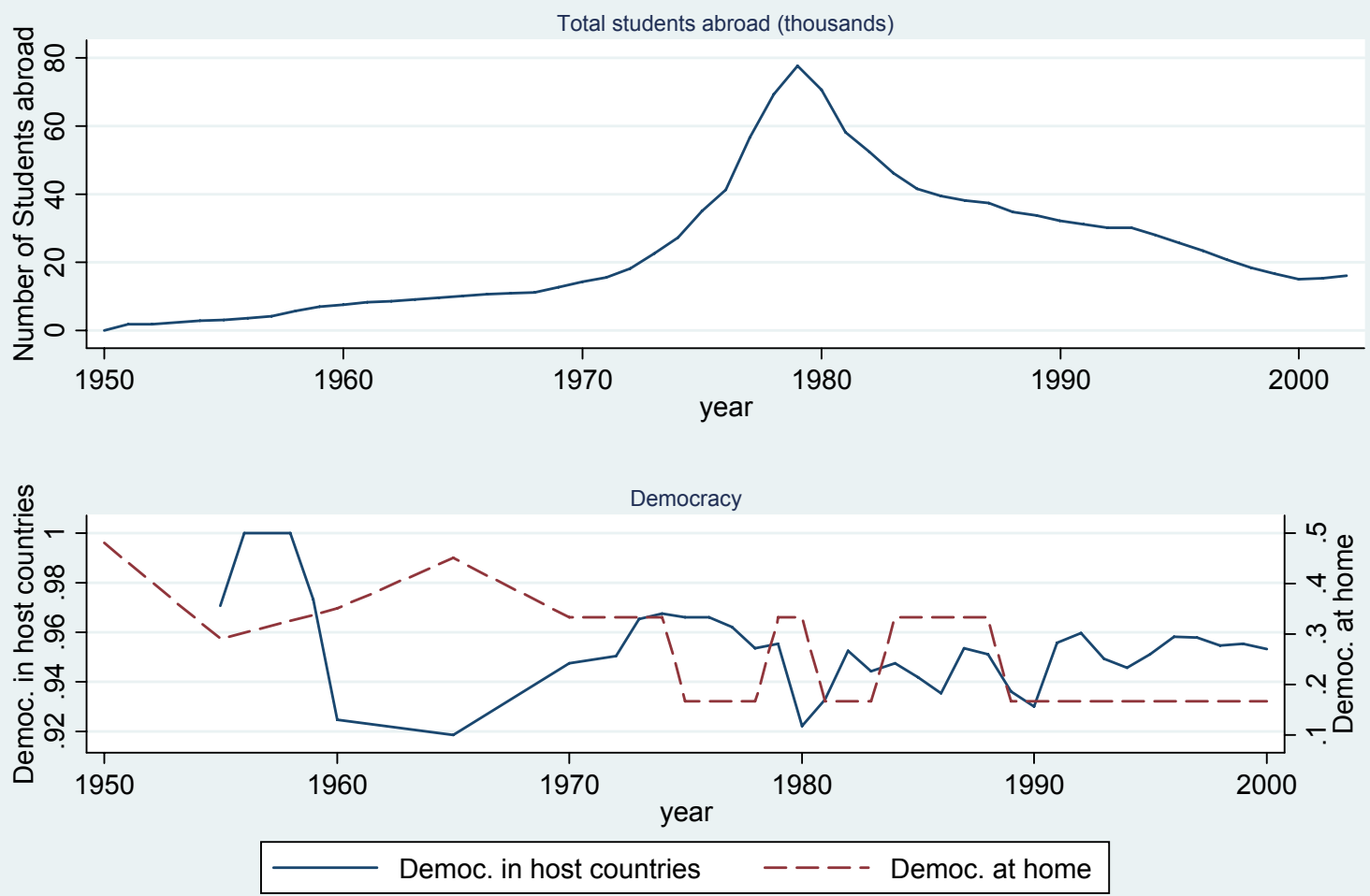

Note: the top panel shows total number of Iranian students abroad in expressed in thousands. The bottom panel shows the level of democracy in home country and in students' host countries, i.e. the average level of democracy in countries where Iranian students are. Both indices are constructed using the Freedom House index of democracy normalized so it lies between 0 and 1 . The construction of the data is described in the data appendix. 


\section{Figure A3. Korea}
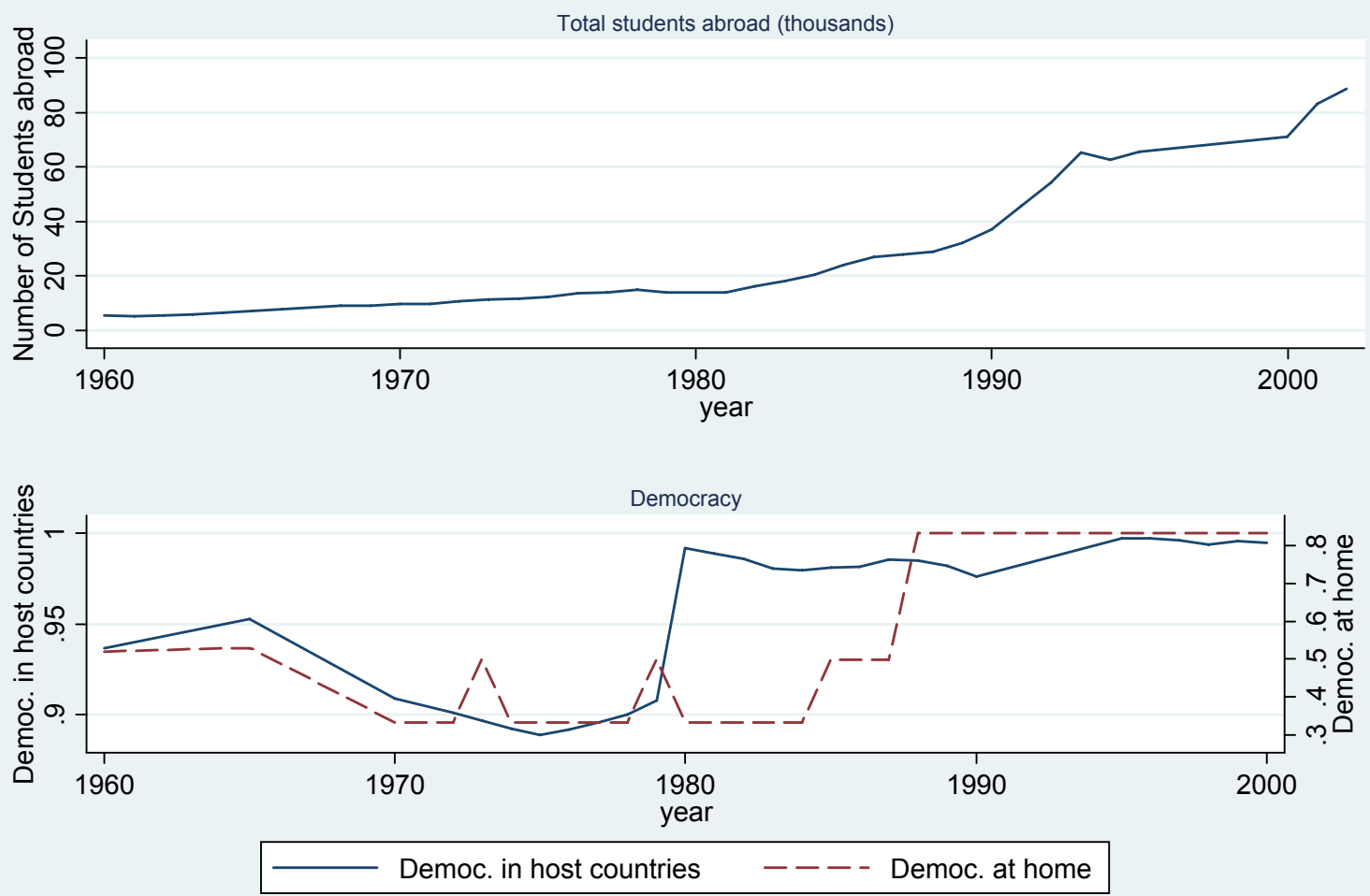

Note: the top panel shows total number of Korean students abroad in expressed in thousands. The bottom panel shows the level of democracy in home country and in students' host countries, i.e. the average level of democracy in countries where Korean students are. Some outliers in the 90 s were excluded in the graph and in the regressions. Both indices are constructed using the Freedom House index of democracy normalized so it lies between 0 and 1 . The construction of the data is described in the data appendix. 
Table A9. Country codes used in Figure A4

\begin{tabular}{|c|c|c|c|c|c|}
\hline Afghanistan & $\mathrm{AFG}$ & Haiti & HTI & Pakistan & PAK \\
\hline Albania & ALB & Honduras & HND & Panama & PAN \\
\hline Argentina & ARG & Hungary & HUN & Paraguay & PRY \\
\hline Australia & AUS & Iceland & ISL & Peru & PER \\
\hline Austria & AUT & India & IND & Philippines & PHL \\
\hline Belgium & BEL & Indonesia & IDN & Poland & POL \\
\hline Bolivia & $\mathrm{BOL}$ & Iran & IRN & Portugal & PRT \\
\hline Brazil & BRA & Iraq & IRQ & Romania & $\mathrm{ROM}$ \\
\hline Bulgaria & BGR & Ireland & IRL & Russia & RUS \\
\hline Cambodia & KHM & Israel & ISR & San Marino & SMR \\
\hline Canada & CAN & Italy & ITA & Saudi Arabia & SAU \\
\hline Chile & CHL & Japan & JPN & Somalia & $\mathrm{SOM}$ \\
\hline China & $\mathrm{CHN}$ & Jordan & JOR & South Africa & ZAF \\
\hline Colombia & COL & Korea & KOR & Spain & ESP \\
\hline CostaRica & CRI & Korea, North & PRK & Sri Lanka & LKA \\
\hline Cuba & CUB & Lao & LAO & Sudan & SDN \\
\hline Cyprus & CYP & Lebanon & LBN & Sweden & SWE \\
\hline Czech Rep. & CZE & Liberia & LBR & Switzerland & $\mathrm{CHE}$ \\
\hline Denmark & DNK & Libya & LBY & Syria & SYR \\
\hline Dominican Rep. & DOM & Luxembourg & LUX & Thailand & THA \\
\hline Ecuador & ECU & Malaysia & MYS & Tunisia & TUN \\
\hline Egypt & EGY & Mexico & MEX & Turkey & TUR \\
\hline El Salvador & SLV & Mongolia & $\mathrm{MNG}$ & UK & GBR \\
\hline Ethiopia & ETH & Morocco & MAR & US & USA \\
\hline Finland & FIN & Nepal & NPL & Uruguay & URY \\
\hline France & FRA & Netherlands & NLD & Venezuela & VEN \\
\hline Germany & DEU & New Zealand & NZL & & \\
\hline Ghana & GHA & Nicaragua & NIC & & \\
\hline Greece & GRC & Nigeria & NGA & & \\
\hline Guatemala & GTM & Norway & NOR & & \\
\hline
\end{tabular}




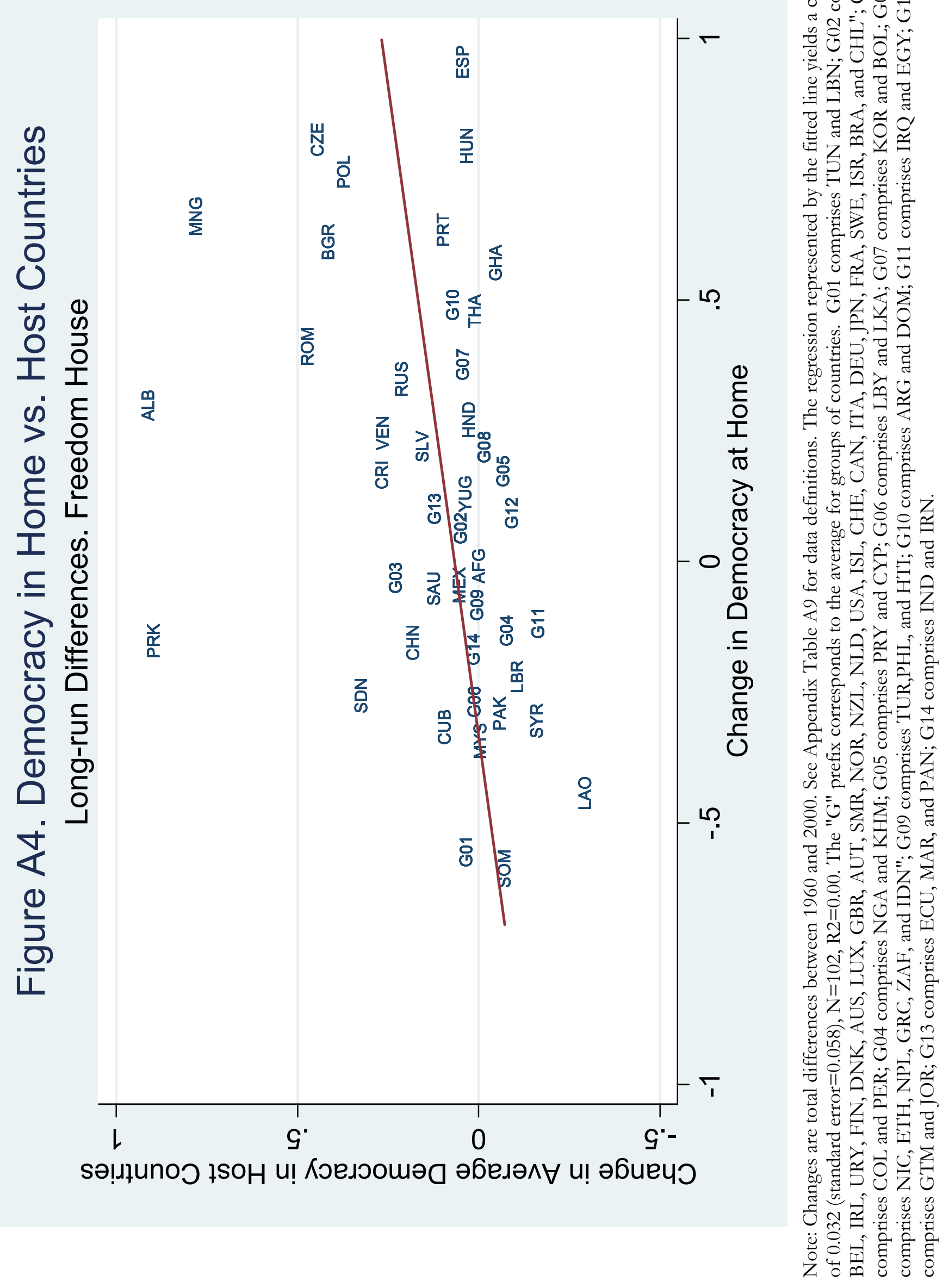

